\title{
Utilização de metilfenidato por usuários do sistema público de saúde em município da região Oeste do Paraná
}

\author{
Utilization of in methylphenidate by users of the public health system in a municipality in \\ western Paraná
}

Maycon Hoffmann Cheffer ${ }^{1}$, Rosa Maria Rodrigues ${ }^{2}$, Solange de Fátima Reis Conterno ${ }^{3}$, Ireni Marilene Zago Figueiredo ${ }^{4}$, Rogério Dias Renovato ${ }^{5}$

\section{RESUMO}

O consumo de metilfenidato tem aumentado a cada ano, e o Brasil é o segundo maior consumidor mundial. Este artigo tem como objetivo caracterizar a utilização de metilfenidato em um município da região Oeste do Paraná, a partir da dispensação do medicamento pela farmácia pública de medicamentos especiais. Estudo descritivo, explicativo, qualitativo com fontes documentais. Metilfenidato foi prescrito com maior frequência ao sexo masculino na faixa etária de sete a 16 anos; psiquiatras e pediatras que mais prescreveram; unidades especializadas e unidades básicas de saúde, as que mais solicitam o medicamento; principais diagnósticos para prescrição de metilfenidato foram TDAH, transtorno de ansiedade generalizada + TDAH e hiperatividade; antidepressivos sertralina e fluoxetina e o antipsicótico risperidona foram os mais dispensadas em conjunto com o metilfenidato; a dose de metilfenidato $10 \mathrm{mg}$ é a mais prescrita; maior frequência de retirada do medicamento foi de um mês, com maior retirada nos meses de agosto, setembro, outubro e novembro. Evidencia-se o aumento dos casos diagnosticados de TDAH e a prescrição do medicamento; contudo, não se pode afirmar que haja o respectivo aumento do consumo, pois a maior frequência de retirada é de um mês, o que mostra descontinuidade ou irregularidade no uso da medicação.

Palavras-chave: Medicalização. Metilfenidato. Transtorno do Déficit de Atenção com Hiperatividade

\section{ABSTRACT}

The consumption of methylphenidate has been increasing every year, and Brazil is the second largest consumer in the world. This article aims to characterize the use of methylphenidate in a municipality in the western region of Paraná, from the dispensation of the drug by the public pharmacy of special drugs. Descriptive, explanatory, qualitative study with documentary sources. Methylphenidate was prescribed more frequently to males in the age group of seven to 16 years; psychiatrists and pediatricians who prescribed more; specialized units and basic health units, the ones that most request the medication; main diagnoses for methylphenidate prescription were ADHD, generalized anxiety disorder + ADHD and hyperactivity; antidepressants sertraline and fluoxetine and the antipsychotic risperidone were the most dispensed together with methylphenidate; the dose of methylphenidate $10 \mathrm{mg}$ is the most prescribed; the highest frequency of medication withdrawal was one month, with the greatest withdrawal in the months of August, September, October and November. There is evidence of an increase in the number of diagnosed ADHD cases and the prescription of the medication; however, it cannot be said that there is an increase in consumption, since the highest frequency of withdrawal is one month, which shows discontinuity or irregularity in the use of medication.
${ }^{1}$ Mestre em Biociências e Saúde. Universidade Estadual do Oeste do Paraná - Unioeste. ORCID: https://orcid.org/0000-0002-93610152

E-mail:

cheffer@hotmail.com

maycon-

2 Doutora em educação. Docente do programa de mestrado em Biociências e Saúde da Universidade Estadual do Oeste do Paraná - Unioeste. ORCID: https://orcid.org/0000-0002-7047$037 \mathrm{X}$

Doutora em educação. Universidade Estadual do Oeste do Paraná - Unioeste. ORCID: https://orcid.org/0000-0003-24938071

4 Doutora em educação. Universidade Estadual do Oeste do Paraná - Unioeste. ORCID: https://orcid.org/0000-0001-88757099

${ }^{5}$ Doutor em educação. Universidade Estadual do Mato Grosso do Sul - UEMS. ORCID: https://orcid.org/0000-0002-55956216

Keywords: Medicalization. Methylphenidate. Attention-Deficit/Hyperactivity Disorder. 


\section{INTRODUÇAOO}

A partir do século $X X$, o consumo de medicamentos aumentou significativamente, devido ao fortalecimento do paradigma biomédico, ao crescimento da indústria farmacêutica, à ampliação do acesso aos medicamentos e à intensificação dos processos de mercantilização da saúde e medicalização da sociedade (POLI NETO; CAPONI, 2007).

A medicalização é o processo por meio do qual os problemas que fazem parte do cotidiano dos indivíduos são transformados em problemas médicos, convertendo questões de origem social e política em questões biológicas, próprias de cada indivíduo (COLLARES; MOYSÉS, 2011). A sociedade atual introduziu como norma "[...] aprimoramento constante e uma necessidade permanente de melhoria de suas capacidades", e isso pode gerar sérios problemas para os indivíduos e para a própria sociedade, ao passo que medicaliza cada vez mais os comportamentos (ORTEGA et al., 2010, p. 508).

Dentre os medicamentos mais utilizadas para tal tem-se o metilfenidato, um agente estimulante do sistema nervoso central, comercialmente divulgado com diferentes nomes, sendo eles: Ritalina $\AA$, Ritalina $L A \circledast$ e Concerta $\AA$, administrados por via oral. "A Ritalina $®$ apresenta-se sob a forma farmacêutica de comprimidos de liberação imediata, enquanto a Ritalina $L A \circledast$, como cápsulas de liberação modificada e o Concerta® como comprimidos de liberação prolongada" (BRATS, 2014, p. 3).

A composição básica da Ritalina® é o cloridrato de metilfenidato e está na classe terapêutica dos psicoestimulantes, utilizado em adultos e crianças acima de seis anos, para tratamento do Transtorno de Déficit de Atenção e Hiperatividade (TDAH), hiperatividade, distúrbios de comportamento e aprendizagem, distractibilidade moderada a grave, déficit de atenção, labilidade emocional, impulsividade, cataplexia e narcolepsia (MOYSÉS, 2012; ITABORAHY; ORTEGA, 2013; SÃO PAULO, 2013; BRATS, 2014; HARAYAMA et al., 2015).

No Brasil, o uso do metilfenidato tem crescido a cada ano (ORTEGA et al., 2010; CALIMAN; DOMITROVIC, 2013, ITABORAHY; ORTEGA, 2013). Em 2000 foram vendidas 70 mil caixas e, em 2010, 2 milhões, fazendo do país o segundo maior consumidor do medicamento mundialmente (MOYSÉS, 2012). Em outubro de 2009 foi registrada a venda de 58.719 caixas, e de 108.609 caixas em outubro de 2013 , aumento de mais de $180 \%$ em quatro anos (HARAYAMA et al., 2015).

Diante da complexidade envolvida na utilização de medicamentos para tratar problemas que nem sempre têm sua causa na dimensão biológica, e considerando a 
proporção com a qual o metilfenidato vem sendo comercializado no Brasil, questiona-se: como o metilfenidato tem sido utilizado em um município no Paraná? O estudo tem como objetivo caracterizar a utilização de metilfenidato no município, a partir da dispensação do medicamento pela farmácia pública de medicamentos especiais, pressupondo que o número de prescrições na realidade estudada tem aumentado, especialmente entre os escolares, em conformidade com dados do cenário nacional.

\section{MATERIAIS E METODOS}

Trata-se de um estudo descritivo explicativo, com fontes documentais, de caráter quantitativo (TRIVINÕS, 2013). O estudo foi realizado em um município localizado na região Oeste do Paraná com uma população estimada de 319.608 habitantes (IBGE, 2017). Os dados são referentes ao período de 2014 a 2016, coletados entre os meses de março e junho de 2017 no arquivo da farmácia de medicamentos especiais da Prefeitura Municipal, pois foi a partir desta data que foram arquivados nessa farmácia, e períodos anteriores a 2014 não estavam acessíveis.

A população de estudo corresponde aos usuários de metilfenidato no município, e a amostra aos usuários de metilfenidato da farmácia de medicamentos especiais do município, caracterizando os usuários e a dispensação via Sistema Único de Saúde (SUS).

As fontes documentais foram os protocolos de solicitação de medicamentos especiais, arquivados no setor da farmácia. Os dados referem ao perfil dos usuários, considerando: gênero, idade, serviço de saúde responsável pelo encaminhamento, diagnóstico, quantidade de medicamento prescrito, quantidade de medicamento dispensado, tempo de uso, medicações associadas e a especialidade médica que prescreveu o medicamento.

Os dados foram sistematizados em planilhas dos programas Microsoft Excel 2013, distribuídos em tabelas de frequências absolutas e relativas, e a análise se amparou na produção científica da área.

O estudo foi aprovado pelo Parecer do Comitê de Ética em Pesquisa nº 2.042.533, em consonância com a Resolução n0466/12 do Conselho Nacional de Saúde que dispõe sobre as questões éticas em pesquisa realizadas com seres humanos. 


\section{RESULTADOS}

O município possuía, em 2017, 36 estabelecimentos públicos de saúde que prescreviam metilfenidato sendo: 17 Unidades Básicas de Saúde (UBS), 13 Estratégia Saúde da Família (ESF) e seis estabelecimentos de saúde especializados.

A farmácia de medicamentos especiais é o único estabelecimento via SUS que dispensa metilfenidato na forma farmacêutica de comprimidos de liberação imediata de 10 $\mathrm{mg}$ cada. O metilfenidato não faz parte da Relação de Medicamentos Essenciais oferecidos pelo SUS e para ser dispensado a farmácia inseriu o medicamento na Relação Municipal de Medicamentos (Remume), por meio da norma operacional $n^{\circ} 44 / 2018$ em que a solicitação de metilfenidato pode ser realizada por médicos da atenção especializada e da atenção básica (CASCAVEL, 2018).

O número de usuários de metilfenidato apresentou crescimento em todos os anos. Eram 113 em 2014, 178 em 2015 e 198 em 2016. O gênero masculino predominou em todos os anos, pois em 2014 foram 100 (88,5\%) masculino e 13 (11,5\%) feminino, em 2015 foram 146 (82\%) masculino e 32 (18\%) feminino e, no ano de 2016, foram 164 (82,8\%) masculino e $34(17,2 \%)$ feminino.

Em 2014, 2015 e 2016, na respectiva ordem, havia 110 (97,3\%), 173 (97,2\%) e 174 $(87,87 \%)$ usuários de metilfenidato com idade inferior a 18 anos e $3(2,7 \%), 5(2,8 \%)$ e 9 (4,5\%), com idade igual ou superior a 18 anos; nesse período, 15 (7,6\%) usuários não tiveram o protocolo encontrado. Em todos os anos, a faixa etária que mais retirou metilfenidato ficou entre sete e 16 anos, 106 (93,8\%) em 2014, 162 (90,9\%), em 2015 e 162 (81,7\%), em 2016.

A especialidade médica que mais prescreveu metilfenidato no ano de 2014 foi a psiquiatria, com 60 (53,1\%) ocorrências, seguida da pediatria, com $30(26,5 \%)$, neurologia e clínica médica com 9 (8\%) ocorrências cada e neuropediatria com 5 (4,4\%). Em 2015, foram $100(56,2 \%)$ da psiquiatria, $37(20,8 \%)$ da pediatria, e $16(9 \%)$ da neurologia, 15 $(8,4 \%)$ da clínica geral e $5(2,8 \%)$ cada para a neuropediatra e especialidade não encontrada. Em 2016, 100 (50,5\%) da psiquiatria, 25 (12,6\%) da neuropediatria, 23 (11,6\%) da pediatria, $20(10,1 \%)$ da neurologia e $15(7,6 \%)$ cada, para a clínica geral e especialidade não encontrada (ausência de registro no/ou protocolo).

Em 2014, 2015 e 2016 as unidades especializadas enviaram solicitações de metilfenidato para a farmácia pública de medicamentos especiais na seguinte proporção: $43(38,5 \%), 99(55,6 \%)$ e 102 (51,5\%), respectivamente, enquanto as UBS solicitaram 39 
(34,5\%), 49 (27,5\%) e 63 (31,8\%), e as USF 31 (27\%), 23 (12,9\%) e $18(9,1 \%)$. Em 2015 e 2016 não foram encontrados 7 (4\%) e 15 (7,6\%).

O diagnóstico clínico, em 2014, 2015 e 2016 são referentes a TDAH 82 (72,6\%), 104 (58,4\%) e 110 (55,5\%); transtorno de ansiedade generalizada + TDAH, 8 (7,1\%), 26 (14,6\%) e 36 (18,2\%); hiperatividade 14 (12,4\%), 17 (9,5\%) e 14 (7,1\%); déficit de atenção 3 (2,6\%), $9(5,1 \%)$ e 9 (4,5\%); item não informado 1 (0,9\%), 13 (7,3\%) e 15 (7,6\%), que corresponde à ausência de registro no/ou protocolo; ansiedade 1 (0,6\%) em 2015 e 4 (2\%) em 2016; e outros diagnósticos: 5 (4,4\%) autismo, atraso desenvolvimento neuropsicomotor, Síndrome de Asperger e transtorno de humor em (2014); 8 (4,5\%) compulsão alimentar, deficiência mental, dificuldade de aprendizagem, dislexia, insônia, transtorno bipolar, microcefalia, Síndrome de Down em 2015 e 10 (5,1\%) agressividade, anoxia neonatal, epilepsia, irritabilidade, hidrocefalia, já faz uso do medicamento, Síndrome de Cornélia de Lange + síndrome alcoólica fetal, Síndrome da Peri-Sylvana + Wester Droyht, transtorno do pânico, transtorno desafiante de oposição em 2016.

Não retiraram outras medicações em associação com o metilfenidato 97 (85,8\%), em 2014, 132 (74,1\%) em 2015 e 144 (72,8\%) em 2016. Dentre as medicações retiradas em associação com o metilfenidato predominou a fluoxetina, com 6 (5,3\%) em 2014, 18 (10,1\%) em 2015 e 22 (11,1\%) em 2016, e a sertralina com 2 (1,7\%) em 2014, 14 (7,9\%) em 2015 e $15(7,6 \%)$ em 2016. A risperidona também foi dispensada em conjunto com o metilfenidato 5 (2,8\%) em 2015 e 4 (2\%) em 2016.

Outras medicações dispensadas em menor proporção com o metilfenidato correspondem a 8 (7,2\%) em 2014, 9 (5,1\%) em 2015 e 13 (6,5\%) em 2016, sendo elas: clonazepan, escitalopran, imipramina, oxcabazepina, valproato de sódio, nortriptilina, fluoxetina + risperidona + sertralina, mirtazapina + venlafaxina, risperidona + amytril e sertralina + bupropiona.

A Tabela 1 revela a posologia diária prescrita de metilfenidato de $10 \mathrm{mg}$, com predomínio de um e dois comprimidos prescritos por dia. 
Tabela 1. Posologia prescrita de metilfenidato, no período de 2014 a 2016

\begin{tabular}{|c|c|c|c|c|c|c|}
\hline \multirow{3}{*}{$\begin{array}{l}\text { Prescrição de comprimidos } \\
\text { (Cp) }\end{array}$} & \multicolumn{6}{|c|}{ Quantidade de usuários } \\
\hline & \multicolumn{2}{|l|}{2014} & \multicolumn{2}{|c|}{2015} & \multicolumn{2}{|c|}{2016} \\
\hline & Quantidade & $\%$ & Quantidade & $\%$ & Quantidade & $\%$ \\
\hline $1 \mathrm{Cp}$ antes da aula & 3 & 2,6 & 5 & 2,8 & 7 & 3,5 \\
\hline 2 Cp antes da aula & & & 1 & 0,6 & 2 & 1 \\
\hline $1 / 2$ Cp por dia & & & 3 & 1,7 & 1 & 0,5 \\
\hline 1 Cp por dia & 60 & 53,1 & 82 & 46,1 & 93 & 46,5 \\
\hline $1 \mathrm{Cp} \mathrm{e} 1 / 2$ ao dia & 6 & 5,3 & 8 & 4,5 & 9 & 4,6 \\
\hline 2 Cp por dia & 39 & 34,5 & 68 & 38,2 & 73 & 36,9 \\
\hline 3 Cp por dia & 3 & 2,7 & 6 & 3,4 & 9 & 4,6 \\
\hline 3 Cp e $1 / 2$ por dia & & & 1 & 0,5 & & \\
\hline 4 Cp por dia & 1 & 0,9 & 2 & 1,1 & 2 & 1 \\
\hline 5 Cp por dia & 1 & 0,9 & 2 & 1,1 & 2 & 1 \\
\hline Total & 113 & 100 & 178 & 100 & 198 & 100 \\
\hline
\end{tabular}

Fonte: Dados coletados na pesquisa (2017).

O gráfico 1 demonstra o número absoluto de usuários que realizaram a retirada mensal de metilfenidato, observando-se maior retirada nos meses de agosto, setembro, outubro e novembro.

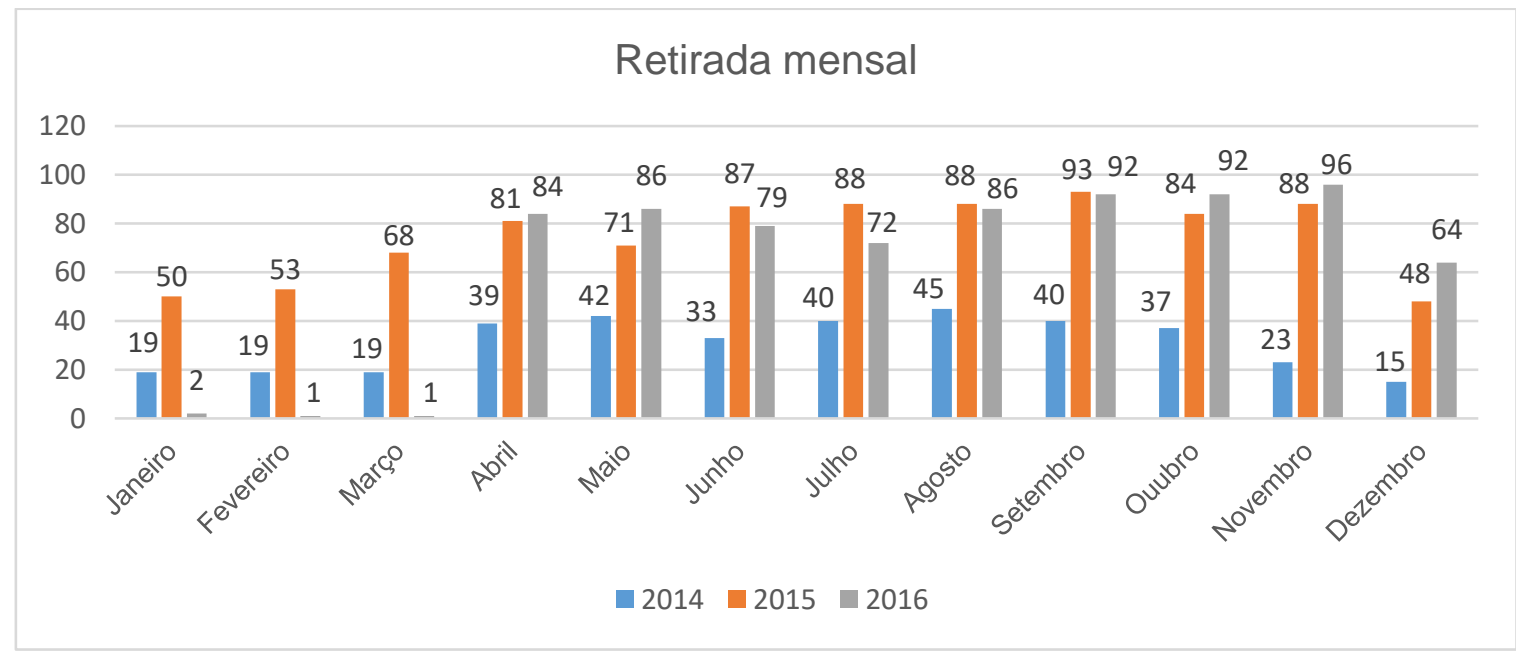

Gráfico 1. Quantidade de usuários que retiraram metilfenidato mensalmente, no período de 2014 a 2016. Fonte: Dados coletados na pesquisa (2017).

Nem todos os usuários retiraram o metilfenidato de acordo com a prescrição. Alguns ficavam meses sem retirar e depois voltavam. Em relação à frequência de retirada do medicamento por mês, em 2014 retiraram um mês 27 (32,9\%); dois 26 (23\%); três 12 (10,6\%); quatro 11 (9,7\%); cinco 15 (13,3\%); seis 13 (11,5\%); sete $3(2,7 \%)$; oito $4(3,5 \%)$; nove e dez 1 (0,9\%) cada. Em 2015 retiraram por um mês 42 (23,6\%); dois 17 (9,6\%); três $11(6,2 \%)$; quatro $22(12,4 \%)$; cinco $9(5,1 \%)$; seis $11(6,2 \%)$; sete $15(8,4 \%)$; oito $15(8,4 \%)$; 
nove 12 (6,7\%); dez 10 (5,6\%); onze e dose 7 (3,9\%) cada. Em 2016 um mês 53 (26,7\%); dois 27 (13,6\%); três 27 (13,6\%); quatro 15 (7,6\%); cinco 21 (10,6\%); seis, sete e oito 17 $(8,6 \%)$ cada e nove $4(2,1 \%)$.

A quantidade de metilfenidato dispensado no ano de 2014 foi de 11.760 comprimidos, em 2015 foram 38.890, e em 2016 foram retirados 29.510 comprimidos.

Foi identificado que usuários, de posse da prescrição, acessavam as unidades de saúde que encaminhavam à farmácia de medicamentos especiais a solicitação de metilfenidato, mas não realizaram a retirada do medicamento após autorização. Dentre eles, observa-se 26 em 2014 dos quais 23 (88,5\%) masculino e 3 (11,5\%) feminino; 23 em 2015, dentre eles 18 (78,3\%) masculino e 5 (21,7\%) feminino e 17 em 2016 dos quais 16 $(94,1 \%)$ masculino e $1(5,9 \%)$ feminino.

\section{DISCUSSÃO}

O aumento de prescrições de metilfenidato tem crescido a cada ano no Brasil (ORTEGA et al., 2010; BRASIL, 2012; ITABORAHY; ORTEGA, 2013). O que se reproduz neste estudo quando foram 113 em 2014, 178 em 2015 e 198 em 2016.

Estudos corroboram a maior incidência de uso do metilfenidato pelo gênero masculino (CAMARGOS JR; NICOLATO, 2009; OCCHI, VIEIRA, PALÁCIO, 2014; MOREIRA et al., 2017). Meninos apresentam elevadas taxas de transtorno de conduta, agressividade, agitação e impulsividade. Em comparação, as meninas apresenta alto nível do transtorno de ansiedade, desatenção e humor; assim, o diagnóstico em meninos é realizado em idade mais precoce (MUSZKAT; MIRANDA; RIZZUTTI, 2011).

A maior frequência dos usuários de metilfenidato esteve na faixa etária de sete a 16 anos. Para Camargos Jr. e Nicolato (2009), a maior frequência de uso de psicoestimulantes está na população de sete a 15 anos. Em Vizotto e Ferraza (2017), o uso do medicamento é prescrito a partir dos 6 anos e possui maior incidência dos 7 aos 8 anos (45\%), período que coincide com o início da escolarização e alfabetização. Em Santos, Oliveira e Bianchini (2018), a faixa etária dos alunos que usaram metilfenidato está entre seis e treze anos.

Ortega et al. (2010), em reportagens acerca do metilfenidato no Brasil, apresentam que em $48,6 \%$ há preocupação em relação ao excesso de diagnósticos médicos e prescrição do medicamento para crianças. Segundo dados da Agência Nacional de Vigilância Sanitária (Anvisa), entre 2009 e 2011, o uso de metilfenidato em crianças com idade de seis a 16 anos aumentou 75\%, especialmente para tratar o TDAH (BRASIL, 2012). 
Não houve neste estudo, usuários de metilfenidato com idade inferior a seis anos, como ocorrido nos estados do Espírito Santo e São Paulo (CALIMAN; DOMITROVIC, 2013; SÃO PAULO, 2013). Da mesma forma, poucos foram os usuários acima de 18 anos, mostrando que o sistema público não tem essa ação como prática.

Segundo Camargos Jr e Nicolato (2009), os pediatras e generalistas são os que mais prescrevem e os quadros mais complexos estão sob a assistência do psiquiatra. Em Perini et al. (2014), a especialidade médica que mais prescreve metilfenidato é o neurologista, psiquiatra e pediatras, seguidos de profissionais de outras especialidades médicas.

Em Vizotto e Ferraza (2017), a psiquiatria, a pediatria e a neuropediatria diante de qualquer demanda ou queixa, prescrevem medicações psicofarmacológicas, e em $97 \%$ dos casos, o pediatra prescreveu na primeira consulta. Em Santos, Oliveira e Bianchini (2018), o perfil de crianças com TDAH está evidente na fala ao apresentarem ansiedade, manias, dificuldade de lidar com frustração, comportamentos antissociais e de origem biológica que melhoram com a administração de medicamentos.

O diagnóstico com maior frequência foi para o TDAH, transtorno de ansiedade generalizada + TDAH e hiperatividade. Quando o metilfenidato surgiu, não havia um diagnóstico específico para seu uso. Os primeiros artigos entre 1937 a 1941 citavam o uso do estimulante para o tratamento da hiperatividade e cefaleia, associado com melhora nos quadros comportamentais e no desempenho escolar das crianças agitadas, tratamento da fadiga presente em quadros psiquiátricos e idosos (ORTEGA et al., 2010; MUSZKAT; MIRANDA; RIZZUTTI, 2011; ESHER; COUTINHO, 2017).

A faixa etária dos usuários e os encaminhamentos para avaliação clínica dos que frequentam instituições educacionais podem revelar uma solicitação de ajuda, devido ao aumento da emissão do diagnóstico de crianças e adolescentes, consideradas portadoras do TDAH, demonstrando necessidade de explicação dos fenômenos que ocorrem no contexto escolar, como se o foco do "problema" estivesse exclusivamente no sujeito (SANTOS; OLIVEIRA; BIANCHINI, 2018).

O diagnóstico do TDAH é complexo, tem alta prevalência em crianças e adolescentes em idade escolar e alta associação de comorbidades psiquiátricas, déficits cognitivos, transtornos invasivos do desenvolvimento e do aprendizado, transtorno desafiador de oposição, transtorno de conduta, transtorno bipolar e depressão, que agravam os quadros de TDAH; desse modo, requerem maior atenção as estratégias de tratamento (PEIXOTO; RODRIGUES, 2008; CALIMAN; DOMITROVIC, 2013; LEONARDO; SUZUKI, 2016). 
O TDAH pode estar associado com a ansiedade de 30 a $40 \%$ e apresentar de 30 a $50 \%$, associação com problemas comportamentais e psiquiátricos como: transtorno desafiador opositivo, transtorno de conduta, transtorno bipolar de humor e ansiedade, tabagismo e abuso de outras substâncias (MUSZKAT; MIRANDA; RIZZUTTI, 2011).

O Transtorno Desafiante de Oposição é um distúrbio característico da infância e possui uma associação com o TDAH, em aproximadamente 15 a $56 \%$ das ocorrências. Em casos associados com o Transtorno de Conduta deve-se usar metilfenidato, e nos mais graves introduzir a risperidona, que tem demostrado eficácia. Quando associados TDAH e ansiedade generalizada, os usuários podem apresentar piora do quadro com o uso de psicoestimulantes, devendo ser associado ao tratamento, intervenções psicoterápicas. $\mathrm{O}$ transtorno bipolar é um transtorno mental grave e sua associação com o TDAH é bastante controversa (MUSZKAT; MIRANDA; RIZZUTTI, 2011).

Em Moreira et al. (2017) estavam associados com o TDAH as comorbidades transtorno de aprendizagem, transtorno de conduta, retardo mental leve, distúrbio de linguagem, ansiedade, epilepsia, transtornos de humor, distúrbios do sistema digestivo, autismo, distúrbios do sistema cardiovascular e respiratório. O metilfenidato com $63,9 \%$ foi a medicação de primeira escolha para tratamento do TDAH. Outras medicações foram associadas concomitante com o metilfenidato, das quais $16,7 \%$ eram antidepressivos (imipramina e fluoxetina), 7,4\% antipsicóticos (risperidona), 1,9\% antiepiléticos (ácido valproico e carbamazepina) e 10,2\% outros. A utilização de antipsicóticos e antiepiléticos exclusivamente para o tratamento de TDAH não foi encontrada na literatura, somente relacionado à presença de outros transtornos associados, como transtorno de ansiedade, de humor e retardo mental moderado.

O antipsicótico risperidona dispensado em conjunto com o metilfenidato $5(2,8 \%)$ em 2015 e 4 (2\%) em 2016, foi utilizado em Vizotto e Ferraza (2017), para 4 (3\%) crianças, para intensificar a "contenção" dos comportamentos incomodativos no espaço escolar. Segundo Nikolov, Jonker e Scahill (2006), a risperidona está empregada no tratamento do autismo, agressão, acessos de ira e automutilação.

A eplepsia pode coexistir com o TDAH por apresentar uma base genética comum e a prevalência da comorbidade ser de três a cinco vezes maior (PEREZ et al., 2010; LOUTIF; CARVALHO, 2010). O diagnóstico de TDAH e epilepsia se apresentam igualmente de maneira confusa e incerta (LEONARDO; SUZIKI, 2016).

No caso do TDAH associado com a epilepsia, o metilfenidato é o medicamento de escolha, apresentando eficácia em curto prazo. Há risco mínimo para aumento das crises 
convulsivas e problemas de atenção ou hiperatividade, que podem ser decorrentes dos problemas psicossociais, mesma propensão genética, participação dos neurotransmissores noradrenalina e dopamina do TDAH e na modulação da excitabilidade neuronal, anormalidades estruturais do cérebro evidenciadas em epilépticos e efeitos colaterais das drogas antiepiléticas (PEREZ et al., 2010; LOUTIF; CARVALHO, 2010). Os antiepiléticos usados em conjunto pelos usuários estudados foram o clonazepan e o valproato de sódio.

Em segunda opção para o tratamento do TDAH podem ser usados os antidepressivos (LOUZA; MATTOS, 2007, MATTOS; ROHDE; POLANCZYK, 2012). Pode-se iniciar simultaneamente com metilfenidato e antidepressivos ou tratar primeiro a depressão com antidepressivo que atue sobre o TDAH. O metilfenidato também tem ação antidepressiva leve (MUSZKAT; MIRANDA; RIZZUTTI, 2011).

Pacientes com TDAH e transtorno bipolar, primeiramente utiliza-se um estabilizador do humor e, posteriormente, o metilfenidato que nunca deve ser utilizado em quadros instáveis. Em pacientes com TDAH e ansiedade, devem ser acrescidos inibidores seletivos da recaptura da serotonina (fluoxetina, sertralina, citalopram, escitalopram), em conjunto ao tratamento com o psicoestimulante (LOUZA; MATTOS, 2007). Segundo Mattos, Rohde e Polanczyk (2012), 30\% dos pacientes com TDAH não reagem aos estimulantes e são tratados com outros medicamentos.

A maior frequência de retirada do medicamento são os meses de agosto, setembro, outubro e novembro. É mais frequente o consumo em períodos letivos, especialmente no segundo semestre (BRASIL, 2012; SÃO PAULO, 2013; ESHER; COUTINHO, 2017).

A maior frequência de retirada do metilfenidato foi de um mês em todos os anos. Segundo Mattos, Rohde e Polanczyk (2012), para estabelecer que um indivíduo está em tratamento contínuo metilfenidato de $10 \mathrm{mg}$, com administração de duas ou três vezes ao dia, por 22 dias por mês, este precisa usar o medicamento durante 10 meses no ano, já que a maioria dos usuários interrompe o uso durante o verão e aos fins de semana.

Os dados evidenciam que a frequência de retirada de metilfenidato por período de dez meses foi $1(0,9 \%)$ em 2014 e 10 (5,6\%) em 2016 e onze e doze meses 7 (3,9\%) cada em 2015 , demonstrando que a retirada via SUS não ocorre de maneira contínua.

A interrupção do uso do medicamento nos finais de semana pode ter indicação em casos em que os sintomas causam prejuízos mais intensos restritos ao ambiente escolar ou para adolescentes em que o controle sobre as drogas ilícitas são mais difíceis nos finais de semana. Já para as pausas durante as férias escolares, pode ocorrer quando o usuário está há cerca de um ano assintomático e com melhora importante da sintomatologia. Desse 
modo, suspende-se o uso da medicação para avaliar a necessidade da continuidade do tratamento (CORREIA FILHO; PASTURA, 2003). Em Moreira et al. (2017), 79,2\% dos usuários de metilfenidato não interromperam o uso do medicamento nos finais de semana.

Em Vizotto e Ferraza (2017), 99\% permaneceram em tratamento por um período de um a quatro anos e, em menor proporção, por um período de cinco a oito anos. Moreira et al. (2017) relatam que $25 \%$ dos usuários tiveram comportamento indicativo de alto grau de adesão, porém, não explicita por quanto tempo, e a baixa adesão foi do tipo não intencional.

A frequência da posologia de metilfenidato prescrito no estudo foi de um comprimido de $10 \mathrm{mg} / \mathrm{dia}$, seguida de dois comprimidos $20 \mathrm{mg} / \mathrm{dia}$, dosagens em conformidade com a literatura que é em média 0,5 a $1 \mathrm{mg} / \mathrm{kg} / \mathrm{dia}$ (ANDRADE; SCHEUER, 2004). Em São Paulo, a dose máxima é 60 mg/dia (SÃO PAULO, 2013). Em Belo Horizonte/MG, a prescrição e a dispensação em 2006, apresentou dosagens superiores a 30 mg/dia (PERINI et al., 2014). No Espírito Santo, o metilfenidato, entre os anos de 2009 e 2011, apresentou expressivo aumento no consumo após a sua dispensação via SUS (CALIMAN; DOMITROVIC, 2013).

O Metilfenidato, quando utilizado por crianças e adolescentes em processo de escolarização, tem seu uso reduzido no período de recesso, finais de semana e férias escolares, mas com consumo crescente ao longo do ano e consequente aumento nas épocas de maior reprovação escolar (BRASIL, 2012; HARAYAMA et al., 2015).

É recomendada a diminuição da dosagem do medicamento nos períodos em que não há atividade escolar, a fim de evitar o consumo abusivo (BRASIL, 2012). Embora o metilfenidato seja eficaz e bem tolerado no tratamento do TDAH (ROCHA; DINIZ; HARA, 2006; ORTEGA et al., 2010), pode causar ou agravar distúrbios psiquiátricos como depressão e ideação suicida, o que pode ter contribuído para o óbito de cinco pacientes no estado de São Paulo (SÃO PAULO, 2013). O medicamento deve ser utilizado com cautela por possuir alto potencial de abuso e dependência (BRATS, 2014).

Os efeitos colaterais mais comuns do metilfenidato são: sonolência, enxaqueca, tontura, lentidão de movimentos, atraso no desenvolvimento, dores abdominais, náuseas, perda de apetite, insônia, irritabilidade, aumento da ansiedade e ganho de peso. Podem ocasionar ainda consequências mais graves como anorexia, taquicardia, hipertensão, psicose, dependência, distúrbios do sistema neurológico como discinesia, espasmos e contrações musculares involuntárias, acidente vascular encefálico, instabilidade emocional, depressão, pânico, hemiplegia, tentativa de suicídio, cefaleia, efeito cardiovasculares, possível diminuição do crescimento, tiques, pesadelos, isolamento social, entre outras graves condições (CORREIA FILHO; PASTURA, 2003; ROCHA; DINIZ; HARA, 2006; 
ORTEGA et al., 2010; ITABORAHY; ORTEGA, 2013; SÃO PAULO, 2013; BRATS, 2014; MOREIRA et al., 2017).

Pode-se problematizar que essa "epidemia" de diagnósticos produz, uma "epidemia" de tratamentos, muitos dos quais altamente prejudiciais à saúde, especialmente nos casos em que não seriam, de fato, necessários. Tal situação tornou-se vantajosa para a indústria farmacêutica, que nos últimos anos tem ampliado seu poder financeiro no campo da saúde (MOYSÉS, 2012; ORTEGA et al., 2010; GOMES; HENNING, 2015).

\section{CONSIDERACÕES FINAIS}

A caracterização da utilização de metilfenidato na realidade estudada confirma o pressuposto de que o número de prescrição do metilfenidato tem aumentado, especialmente entre os escolares.

A farmácia pública de medicamentos especiais precisa elaborar propostas para dispensação do metilfenidato como forma de racionalizar o seu uso, uma vez que não estão claros os critérios diagnósticos de todos os usuários, bem como a continuidade e a evolução do seu tratamento, o que remete a questionamentos, sobretudo em razão da baixa frequência de retirada do medicamento e dos possíveis efeitos fisiológicos pelo uso a curto prazo ou prolongado da medicação.

Diante da necessidade de respostas, novas pesquisas são necessárias a fim de executar as recomendações do Ministério da Saúde para a adoção de práticas não medicalizantes, bem como explicar como que, diante do crescente número de usuários de metilfenidato no ano de 2016, a quantidade de comprimidos dispensados foi menor que no ano de 2015 em que apresentava uma quantidade inferior de usuários; assim como caracterizar a origem das queixas pelas quais os usuários são encaminhados para consulta médica, seriam oriundas da escola, professores ou familiares?

\section{REFERÉNCIAS}

ANDRADE, E. R.; SCHEUER, C. Análise da eficácia do metilfenidato usando a versão abreviada do questionário de conners em transtorno de déficit de atenção/hiperatividade. Arq. Neuro-Psiquiatr, São Paulo, v. 62, n. 1, p. 81-85, mar. 2004. Disponível em: $<$ https://www.scielo.br/scielo.php?pid=S0004-

282X2004000100014\&script=sci_abstract\&tlng=pt>. Acesso em: 04 set. 2020.

BRASIL. Agência Nacional de Vigilância Sanitária. Boletim de Farmacoepidemiologia. Prescrição e consumo de metilfenidato no Brasil: identificando riscos para o 
monitoramento e controle sanitário. Brasília, ano 2, n. 2, jul./dez., 2012. Disponível em: <https://www.anvisa.gov.br/sngpc/boletins/2012/boletim_sngpc_2_2012_corrigido_2.pdf>. Acesso em: 5 jun. 2021.

BRATS. Boletim Brasileiro de Avaliação de Tecnologias em Saúde. Metilfenidato no tratamento de crianças com transtorno de déficit de atenção e hiperatividade. Brasília, 2014. Disponível em: <https://docs.bvsalud.org/biblioref/2021/04/1178586/brats23.pdf>. Acesso em: 10 jun. 2021.

CALIMAN, L. V.; DOMITROVIC, N. Uma análise da dispensa pública do metilfenidato no Brasil: o caso do Espírito Santo. Physis, Rio de Janeiro, v. 23, n. 3, p. 879-902, set. 2013. Disponível em: <https://www.scielo.br/scielo.php?pid=S010373312013000300012\&script=sci_abstract\&tlng=pt>. Acesso em: 12 abr. 2021.

CAMARGOS JR. W.; NICOLATO, R. Características das prescrições no transtorno de déficit de atenção/hiperatividade. J. bras. psiquiatr, Rio de Janeiro, v. 58, n. 3, p. 195-199, 2009. Disponível em: <https://www.scielo.br/scielo.php?script=sci_arttext\&pid=S004720852009000300009>. Acesso em: 12 abr. 2021.

CASCAVEL. Prefeitura Municipal de Cascavel, Paraná. Norma Operacional $n^{\circ}$ 44/2018 da Divisão de assistência farmacêutica sobre o protocolo de solicitação de medicamentos especiais, Cascavel, 2018. Disponível em: <http://irsas.cascavel.pr.gov.br/arquivos/19022018_ci_020_2018_protocolo_de_solicita cao_de_medicamentos_especiais.pdf>. Acesso em: 11 out. 2020.

COLLARES, C. A. L.; MOYSÉS, M. A. A. Preconceitos no cotidiano escolar: ensino e medicalização. Campinas: Cortez, 2011.

CORREIA FILHO, A. G. C.; PASTURA, G. ROHDE. As medicações estimulantes. In: RHODE, L. A.; MATTOS, P. (Orgs.) Princípios e práticas em TDAH. Porto Alegre: Artemed, 2003. Ap. 161-173.

ESHER, A.; COUTINHO, T. Uso racional de medicamentos, farmaceuticalização e usos do metilfenidato. Ciênc. saúde coletiva, Rio de Janeiro, v. 22, n. 8, p. 2571-2580, 2017. Disponível em: <https://www.scielo.br/scielo.php?pid=S141381232017002802571\&script=sci_abstract\&tlng=pt>. Acesso em: 10 nov. 2020.

GOMES, R. K.; HENNING, F. A medicalização da infância e o crescimento do uso de psicofármacos por crianças no Brasil. Revista de Extensão e Iniciação Científica Unisociesc, Curitiba, v. 2, n. 1, p. 13-30, 2015. Disponível em: <http://reis.unisociesc.com.br/index.php/reis/article/view/23/26>. Acesso em: 3 jan. 2021.

HARAYAMA, R. et al. Fórum sobre medicalização da educação e da sociedade. Nota técnica: o consumo de psicofármacos no Brasil, dados do sistema nacional de gerenciamento de produtos controlados Anvisa (2007-2014). p. 2015-2025. Brasil, 2015. Disponível em: <http://medicalizacao.org.br/wpcontent/uploads/2015/06/NotaTecnicaForumnet_v2.pdf>. Acesso em: 13 ago. 2020.

IBGE. Instituto Brasileiro de Geografia e Estatística. Ensino - matrículas, docente e rede escolar. 2017. Disponível em: <https://cidades.ibge.gov.br/brasil/pr/cascavel/panorama 2 . Acesso em: 2 out. 2017. 
ITABORAHY, C.; ORTEGA, F. O metifenidato no Brasil: uma década de publicações. Ciênc. saúde coletiva, Rio de Janeiro, v. 18, n. 3, p. 803-816, mar. 2013. Disponível em: $<$ https://www.scielo.br/scielo.php?pid=S1413-

81232013000300026\&script=sci_abstract\&tIng=pt>. Acesso em: 12 abr. 2020.

LEONARDO, N. S. T.; SUZUKI, M. A. Medicalização dos problemas de comportamento na escola: perspectivas de professores. Fractal, Rev. Psicol, Rio de Janeiro, v. 28, n. 1, p. 4654, abr. 2016. Disponível em: <https://www.scielo.br/scielo.php?pid=S198402922016000100046\&script=sci_abstract\&tIng=pt>. Acesso em: 12 abr. 2021.

LOUTFI, K. S.; CARVALHO, A. M. Possíveis interfaces entre TDAH e epilepsia. J. bras. Psiquiatr, Rio de Janeiro, v. 59, n. 2, p. 146-155, 2010. Disponível em: $<$ https://www.scielo.br/scielo.php?pid=S0047-

$20852010000200011 \&$ script=sci_abstract\&tlng=pt>. Acesso em: 13 nov. 2020.

LOUZA, M. R.; MATTOS, P. Questões atuais no tratamento farmacológico do TDAH em adultos com metilfenidato. J. bras. psiquiatr, Rio de Janeiro, v. 56, supl. 1, p. 53-56, 2007. Disponível em: <https://www.scielo.br/scielo.php?script=sci_arttext\&pid=S004720852007000500012>. Acesso em: 12 abr. 2021.

MATTOS, P.; ROHDE, L. A.; POLANCZYK, G. V. O TDAH é subtratado no Brasil. Rev. Bras. Psiquiatr, São Paulo, v. 34, n. 4, p. 513-514, dez. 2012. Disponível em: $<$ https://www.scielo.br/scielo.php?pid=S1516-

44462012000400023\&script=sci_arttext\&tlng=pt>. Acesso em: 12 abr. 2021.

MOREIRA, M. T. et al. Transtorno de déficit de atenção/hiperatividade: prevalência e uso de psicofármacos em crianças de um ambulatório no Sul de Santa Catarina. Arquivos Catarinenses de Medicina, Florianópolis/SC, v. 46, n. 3, p. 106-117, set. 2017. Disponível em: <http://www.acm.org.br/acm/seer/index.php/arquivos/article/view/312>. Acesso em: 02 nov. 2020.

MOYSÉS, M. A. A. Não às drogas da obediência. Metrópole, Campinas, p. 10-11, 2012. Entrevista concedida a Karina Fusco. Disponível em: <http://medicalizacao.org.br/nao-asdrogas-da-obediencia/>. Acesso em: 20 maio 2021.

MUSZKAT, M.; MIRANDA, M.C.; RIZZUTTI, S. Transtorno do déficit de atenção e hiperatividade. 3. vol. São Paulo: Coleção Educação e Saúde, 2011.

NIKOLOV, R.; JONKER, J.; SCAHILL, L. Autismo: tratamentos psicofarmacológicos e áreas de interesse para desenvolvimentos futuros. Rev. Bras. Psiquiatr, São Paulo, v. 28, supl. 1, p. 39-46, May 2006.2 Disponível em: <https://www.scielo.br/scielo.php?script=sci_arttext\&pid=S1516-44462006000500006>. Acesso em: 25 out. 2020.

OCCHI, N. L. O.; VIEIRA, G.L.; PALÁCIO, S. G. Efeitos do metilfenidato no desempenho motor em escolares entre 7 e 10 anos com transtorno de déficit de atenção e hiperatividade. UneCesumar, VII Mostra Interna de Trabalhos de Iniciação Científica, Maringá/PR, out. 2014. 
ORTEGA, F. et al. Ritalina no Brasil: produção, discurso e práticas. Interface - Comunic., Saude, Educ, Botucatu, v. 14, n. 34, p. 499-510, jul./set, 2010. Disponível em: <https://www.scielosp.org/article/icse/2010.v14n34/499-512/>. Acesso em: 10 jun. 2020.

PEIXOTO, A. L. B.; RODRIGUES, M. M. P. Diagnóstico e tratamento de TDAH em crianças escolares, segundo profissionais da saúde mental. Aletheia, Canoas, n. 28, p. 91-103, dez. 2008. Disponível em <http://pepsic.bvsalud.org/scielo.php?script=sci_arttext\&pid=S141303942008000200008>. Acesso em: 3 out. 2020.

PEREZ, E. B. et al. Comorbilidad trastorno por déficit de atención con hiperactividad (TDAH) y epilepsia. REV MED HONDUR, México, v. 78, n. 2, p. 83-90, 2010. Disponível em: <https://revistamedicahondurena.hn/assets/Uploads/Vol78-2-2010-9.pdf>. Acesso em: 12 abr. 2021.

PERINI, E. et al. Prescription, dispensation and marketing patterns of methylphenidate. Rev. Saúde Pública, São Paulo, v. 48, n. 6, p. 873-880, Dec. 2014. Disponível em: $<$ https://www.scielo.br/j/rsp/a/bMQcRvXfQjCR83cqSg8TDks/?lang=en>. Acesso em: 5 set. 2020.

POLI NETO, P.; CAPONI, S. N. C. A medicação da beleza. Interface - Comunic., Saude, Educ, v. 11, n. 23, p. 569-84, set./dez. 2007. Disponível em: <http://www.scielo.br/pdf/icse/v11n23/a12v1123.pdf>. Acesso em: 26 jun. 2020.

ROCHA, F. L.; MALLOY-DINIZ, L. F.; HARA, C. Emprego de metilfenidato para o tratamento de déficit cognitivo em paciente com sequela de traumatismo cranioencefálico. J. bras. Psiquiatr, Rio de Janeiro, v. 55, n. 1, p. 78-81, 2006. Disponível em: $<$ https://www.scielo.br/scielo.php?script=sci_arttext\&pid=S0047-

20852006000100012\#: :text=0\%20uso\%20de\%20metilfenidato\%20mostrou,dois\%20ano s\%20ap\%C3\%B3s\%20o\%20acidente.>. Acesso em: 12 abr. 2021.

SANTOS, R. L.; OLIVEIRA, F. N.; BIANCHINI, L. G. B. Medicalização da aprendizagem e resiliência: significações produzidas na escola. Revista Ibero-Americana de Estudos em Educação, v. 13, n. 4, p. 1792-1813, out./dez. 2018. Disponível em: <https://periodicos.fclar.unesp.br/iberoamericana/article/view/10190>. Acesso em: 06 nov. 2020.

SÃO PAULO. Secretaria de Estado da Saúde de São Paulo. Núcleo de Farmacovigilância do Centro de Vigilância Sanitária da Secretaria de Estado da Saúde de São Paulo. Alerta terapêutico em farmacovigilância 01/2013 - Metilfenidato: indicações terapêuticas e reações adversas. São Paulo, 2013. Disponível em: $<$ http://www.cvs.saude.sp.gov.br/zip/E CM CVS-045 020813.pdf>. Acesso em: 30 out. 2020.

TRIVIÑOS, A. N. S. Introdução à pesquisa em ciências sociais: a pesquisa qualitativa em educação. 1. ed. 22. reimpr. São Paulo: Atlas, 2013.

VIZOTTO, L. P.; FERRAZZA, D. A. A infância na berlinda: sobre rotulações diagnósticas e a banalização da prescrição de psicofármacos. Estud. psicol. (Natal), Natal, v. 22, n. 2, p. 214-224, jun. $2017 . \quad$ Disponível em: <http://pepsic.bvsalud.org/scielo.php?script=sci_arttext\&pid=S1413294X2017000200010>. Acesso em: 13 nov. 2020. 